\title{
An Effective Approach to Enhance Centroid Classifier for Text Categorization
}

\author{
Songbo Tan and Xueqi Cheng \\ Information Security Center, Institute of Computing Technology, China \\ tansongbo@software.ict.ac.cn, tansongbo@gmail.com
}

\begin{abstract}
Centroid Classifier has been shown to be a simple and yet effective method for text categorization. However, it is often plagued with model misfit (or inductive bias) incurred by its assumption. To address this issue, a novel Model Adjustment algorithm was proposed. The basic idea is to make use of some criteria to adjust Centroid Classifier model. In this work, the criteria include training-set errors as well as training-set margins. The empirical assessment indicates that proposed method performs slightly better than SVM classifier in prediction accuracy, as well as beats it in running time.
\end{abstract}

Keywords: Text Categorization, Information Retrieval, Data Mining.

\section{Introduction}

With the rapid growth of texts in the Internet, text categorization has received more and more attention in information retrieval community. Numerous machine learning approaches have been introduced to deal with text classification, including Centroid Classifier [1], Naive Bayes [2], Winnow or Perceptron [3], Voting [4] and Support Vector Machines (SVM) [5].

Despite simplicity and straightforwardness, Centroid Classifier has proved to be an effective and yet robust method for text categorization. However, it is often plagued with inductive bias [6] or model misfit [7]. For example, Centroid Classifier makes a simple assumption that a given document should be assigned a particular class if the similarity of this document to the centroid of the class is the largest. However, this supposition is often violated when there exists a document from class $A$ sharing more similarity with the centroid of class $B$ than that of class $A$. The more serious the model misfit is, the poorer the classification performance will be.

In this work, we proposed a novel Model Adjustment (MA) algorithm to cope with model misfit problem of Centroid Classifier. The basic idea is to pick out some training examples to adjust Centroid Classifier model. The most popular and simple method is to select misclassified examples. From the perspective of machine learning, however, low training-error-rate does not indicate low error-rate for unseen examples. To address this issue, another measure, i.e., training-set margin, was incorporated. In other words, misclassified examples as well as small-margin examples were picked out to update the classifier model. 
The experimental results show that proposed technique is able to improve classification performance of Centroid Classifier significantly. Furthermore, the resulting classifier performs slightly better than SVM classifier in classifying accuracy, as well as beats it in running time.

\section{Related Work}

In this section, we briefly review the related researches and compare them with proposed method.

Voting [4] is a famous strategy for correction of inductive bias. It works by taking a classifier and training set as input and training the classifier multiple times on different versions of the training set. Compared to this method, MA has three particularities. First, MA does not need to retrain the classifier multiple times on the different versions of the entire training set. Second, MA produces only one refined classifier. Furthermore, Voting utilizes only training-set errors while MA employs training-set errors as well as training-set margins.

A "dragpushing" strategy for Centroid and Naïve Bayes Classifier is proposed by Tan et al[8]. This method takes advantage of misclassified training examples to successively refine classification model by online-modification. Different from this method, MA employs training error as well as training margin.

The weight adjustment scheme [1] is to use a measure of the discriminating power of each term to gradually adjust the weights of all features concurrently. In contrast to this scheme, MA employs training error and training margin rather than discriminating power as adjustment criteria.

\section{Centroid Classifier}

The idea behind the centroid classification algorithm [1] is extremely simple and straightforward. First we compute the weighted representation of each training document using normalized TFIDF [4]. Then we calculate the prototype vector or centroid vector $C_{i}$ for each training class $c_{i}$ :

$$
C_{i}=\frac{1}{\left|c_{i}\right|} \sum_{d \in c_{i}} d .
$$

where $|z|$ denotes the cardinality of set $z$.

Thirdly we calculate the similarity of one document $d$ to each centroid by innerproduct measure,

$$
\operatorname{Sim}\left(d, C_{i}\right)=d \cdot C_{i} \cdot
$$

Lastly, based on these similarities, we assign $d$ the class label corresponding to the most similar centroid. 


\section{Proposed Technique}

In this section we first illustrate the rationale to deal with inductive bias and then present the detailed algorithm of proposed method.

Let us take a two-class text data as an example. The data distribution is illustrated as Fig 1. Class " $A$ " spread as grey is elliptically populated; while class " $B$ " packed as white is roundly distributed. $C_{A}$ and $C_{B}$ are the centroids of class $A$ and class $B$ respectively. Middle Line is the perpendicular bisector of the line between $C_{A}$ and $C_{B}$. From another perspective, Middle Line serves as a decision hyper-plane that separate class $A$ and class $B$. Obviously, the examples of category $A$ on the right of Middle Line share more similarity with centroid $C_{B}$ rather than $C_{A}$, so they will be misclassified into class $B$. This is the case that the supposition of Centroid Classifier is violated by data distribution.

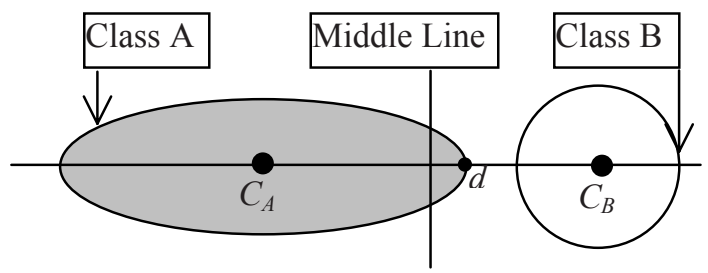

Fig. 1. The outline of Original Centroids

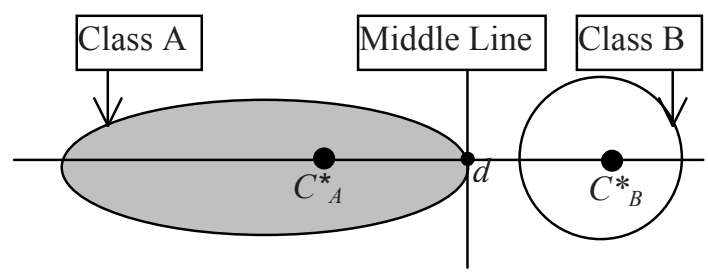

Fig. 2. The outline of Refined Centroids

In order to reduce this model bias, we make use of training errors to adjust its prototype vectors. For example, if document $d$ of class $A$ is misclassified into class $B$, both centroid $C_{A}$ and $C_{B}$ should be moved right by the following formulas (3) and (4) respectively,

$$
\begin{aligned}
& C_{A}^{*}=C_{A}+\eta \cdot d . \\
& C_{B}^{*}=C_{B}-\eta \cdot d .
\end{aligned}
$$

where $\eta$ denotes the "LearnRate" that is used to control the strength of update. With this so-called move operation, $C_{A}$ and $C_{B}$ are both moving right gradually. At the end of this kind of move operation (see Fig 2), no example of class $A$ locates at the right of Middle Line so no example will be misclassified. 
However, above adjustment approach employs only one criterion, i.e., training-set error. From the point of view of machine learning, training-set error based method cannot guarantee the generalization capability of base classifiers for unseen examples. To fully demonstrate this problem, we revisit the aforementioned two-class dataset. Without loss of generality, we can construct the future distribution of class $A$ and class $B$. Obviously the training examples are only a small portion of unseen examples of class $A$ and class $B$ (as illustrated Fig 3). The unseen examples of class $A$ are denoted by "." or grey; the unseen examples of class $B$ are denoted by "-" or white.

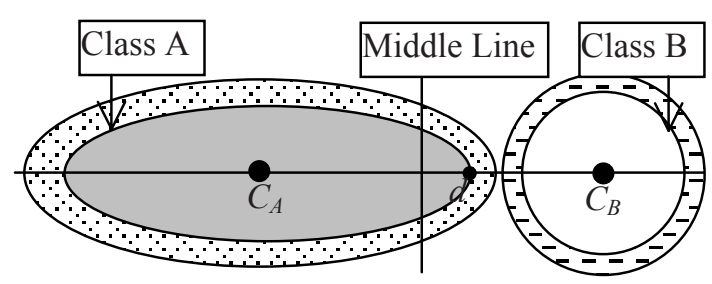

Fig. 3. The distribution of unseen examples of Class A and Class B

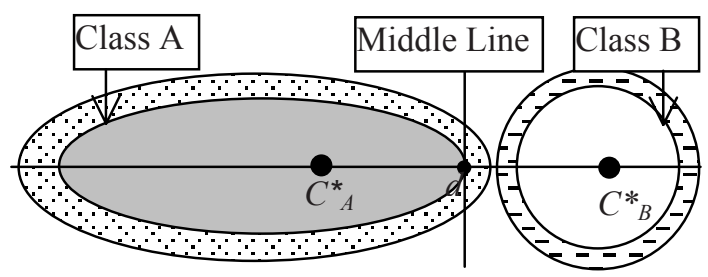

Fig. 4. Refining the centroids by training examples

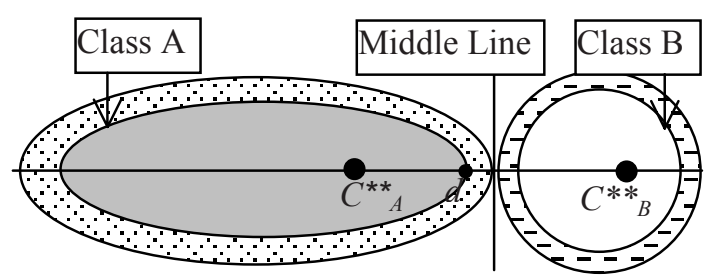

Fig. 5. Refining the centroids by training examples and unseen examples

After adjusting classifier model by misclassified training examples, the Middle Line moves right to the border of class $A$ (see Fig. 4). In this case, all training examples can be correctly classified, but not all unseen examples can be correctly classified. For example, we can observe that the unseen examples of class $A$ on the right of Middle Line will be misclassified into class $B$. This observation indicates that training-set 
error based model update cannot guarantee the classification performance of base classifiers for unseen documents.

With the aim to improve the classification ability of classifier for unseen examples, the Middle Line should be moved right again. That is to say, centroid $C_{A}$ and $C_{B}$ should be both moved right. To accomplish this goal, some correctly classified examples near Middle Line in class $A$ should be employed to adjust centroid $C_{A}$ and $C_{B}$. That is, for each training example in class $A$, we not only require its $\operatorname{Sim}\left(d, C_{A}\right)$ is bigger than $\operatorname{Sim}\left(d, C_{B}\right)$; but also demand $\operatorname{Sim}\left(d, C_{A}\right)$ exceeds $\operatorname{Sim}\left(d, C_{B}\right)$ by a wide margin.

To further illustrate this kind of margin (denoted by $\rho$ ), we take document $d$ in Fig 4 as an example. Although document $d$ can be correctly classified because the Middle Line has moved to the border of class $A$, its margin is very close to zero since it lies exactly on the Middle Line. Hence in order to enlarge the margin, both centroid $C_{A}$ and $C_{B}$ should be moved right again by formulas (3) and (4) respectively. After a number of this kind of moving operations, the Middle Line moves to the border of unseen examples of class $A$ (as demonstrated in Fig 5). In this case, all unseen examples can be correctly categorized. This is the mechanism that margin can boost the classification ability of classifier for unseen examples.

To concentrate our attention on small-margin examples, we introduce a small positive margin threshold, MinMargin (denoted by $\theta$ ). If the margin of document $d$ is smaller than MinMargin, it should be employed to adjust the classifier model. Furthermore, to balance training errors and training margins, we introduce a constant parameter "Weight" (denoted by $\omega$ ). As a result, we can write down the batch-update formula as following,

$$
C_{A}^{*}=C_{A}+\eta \times\left\{\sum_{\substack{d \in c_{A} \\ \rho(\mathrm{d}<0}} d-\sum_{\substack{d \neq c_{A} \\ \rho(\mathrm{d})<0}} d+\omega \times\left(\sum_{\substack{d \in c_{A} \\ 0<\rho(\mathrm{d}) \theta}} d-\sum_{\substack{d \neq c_{A} \\ 0<\rho(\mathrm{d}) \theta \theta}} d\right)\right\} .
$$

The detailed algorithm is presented in Fig 6. First it loads training data and parameters (including MaxIteration, Weight and LearnRate), and then calculates one centroid for each category. In one iteration of the updating phase, it needs to categorize all training documents, and then makes use of these misclassified examples and small-margin examples to adjust centroids by formula (5). For the sake of brevity, we refer to the model-adjustment algorithm as MA.

1 Load training data and parameters;

2 Calculate $\mathrm{C}_{i}$ for each class $\mathrm{C}_{i}$;

3 For iter=1 to Maxiteration Do

3.1 Classify all training documents;

3.2 Update centroids by formula (5);

Fig. 6. The outline of Model Adjustment for Centroid Classifier 


\section{Empirical Assessment}

\subsection{Experimental Design}

In our experiment, we use five corpora: Reuters- $21578^{1}, 20$ NewsGroup $^{2}$, Industry Sector $^{3}$, OHSUMED ${ }^{4}$ and RCV1 ${ }^{5}$. For Reuters-21578, we used its subset: one consisting of 92 categories and in total 10,346 documents; for 20NewsGroup, we use a subset consisting of total categories and 19,446 documents; for Industry Sector, we use a subset called as Sector-48 consisting of 48 categories and in all 4,581 documents; for OHSUMED, we use a subset (called ohscal ${ }^{6}$ in [1]) that contains 11,162 documents and in total 10 categories; for RCV1, we use a subset consisting of 56 categories and 41,320 documents. Typically, we use 2/3 of documents for training and $1 / 3$ for testing; exceptionally, in order to reduce the training time for RCV1, we use $10 \%$ of its total documents for training and $90 \%$ for testing.

To evaluate the performance of a text classifier, we use MicroF1 and MacroF1 scores [4]. To remove redundant features and save running time, we employ Information Gain as feature selection method. Algorithms are coded in $\mathrm{C}++$ and running on a Pentium-4 machine with a single 3.0GHz CPU and 512M memory. For SVM classifier, we employed LibSvm that can directly deal with multi-class classification problems. (www.csie.ntu.edu.tw/ cjlin/). We left all parameters as default. For Winnow classifier, we run Balanced Winnow [3]. The promotion parameter $\alpha$ and the demotion $\beta$ (learning rates) were fixed as 1.2 and 0.8 respectively.

\subsection{Comparison with Other Methods}

Table 1 shows performance comparison in MicroF1 and MacroF1. Feature number is set to 10,000; For MA, MaxIteration, Weight, LearnRate, and MinMargin are set to $10,0.2,0.5$, and 0.1 respectively. From this table, we can see that MA improves the performance of Centroid Classifier dramatically, and the improvement is especially significant on Sector-48. For example, MA improves Centroid Classifier by about $9 \%$ on Sector-48. In a word, Model Adjustment is an effective and robust method to boost the performance of Centroid Classifier.

MA outperforms all the other methods on OHSUMED, Reuters, Sector-48 and RCV1. Especially on Reuters, the MicroF1 of MA is one percent lower than LibSvm but its MacroF1 is 12 percent higher than LibSvm. In total MA performs slightly better than LibSvm. Consequently we can say that MA is an efficient and competitive algorithm for text classification.

Table 2 reports the training time of five methods on five text collections. Feature number is set to 10,000; For MA, MaxIteration, Weight, LearnRate, and MinMargin are set to $10,0.2,0.5$, and 0.1 respectively. As we can observe from this table, the

\footnotetext{
${ }_{1}^{1}$ http://www.daviddlewis.com/resources/testcollections/reuters21578/.

2 http://www-2.cs.cmu.edu/afs/cs/project/theo-11/www/wwkb.

3 http://www-2.cs.cmu.edu/afs/cs.cmu.edu/project/theo-20/www/data/.

$4 \mathrm{ftp}: / /$ medir.ohsu.edu/pub/OHSUMED/.

5 http://www.daviddlewis.com/resources/testcollections/rcv1/.

${ }^{6} \mathrm{http}: / /$ www.cs.umn.edu/ han/data/tmdata.tar.gz.
} 
Table 1. The performance of different methods

\begin{tabular}{c|c|c|c|c|c|c|c|c|c|c}
\hline & \multicolumn{2}{|c|}{ MA } & \multicolumn{2}{c|}{ Centroid } & \multicolumn{2}{c|}{ Winnow } & \multicolumn{2}{c|}{ Perceptron } & \multicolumn{2}{c}{ LibSvm } \\
\cline { 2 - 11 } & MicroF1 & MacroF1 & MicroF1 & MacroF1 & MicroF1 & MacroF1 & MicroF1 & MacroF1 & MicroF1 & $\begin{array}{c}\text { MacroF } \\
\mathbf{1}\end{array}$ \\
\hline OHSUMED & $\mathbf{0 . 8 0 4 9}$ & $\mathbf{0 . 7 9 4 0}$ & 0.7676 & 0.7600 & 0.7193 & 0.7110 & 0.7100 & 0.6996 & 0.7906 & 0.7800 \\
\hline Reuters & 0.8565 & $\mathbf{0 . 6 0 6 1}$ & 0.7820 & 0.5617 & 0.8263 & 0.4891 & 0.7918 & 0.4553 & $\mathbf{0 . 8 6 9 4}$ & 0.4875 \\
\hline Sector-48 & $\mathbf{0 . 8 9 7 0}$ & $\mathbf{0 . 9 0 0 0}$ & 0.8055 & 0.8152 & 0.8003 & 0.8389 & 0.7845 & 0.7943 & 0.8732 & 0.8780 \\
\hline NewsGroup & 0.8892 & 0.8859 & 0.8429 & 0.8389 & 0.8105 & 0.8161 & 0.8089 & 0.8081 & $\mathbf{0 . 9 0 4 0}$ & $\mathbf{0 . 9 0 2 9}$ \\
\hline RCV1 & 0.7166 & $\mathbf{0 . 4 9 1 2}$ & 0.6778 & 0.4883 & 0.6142 & 0.4138 & 0.6335 & 0.3379 & $\mathbf{0 . 7 2 1 3}$ & 0.4126 \\
\hline
\end{tabular}

Table 2. Training Time in seconds

\begin{tabular}{c|c|c|c|c|c}
\hline & MA & Centroid & Winnow & Perceptron & LibSvm \\
\hline OHSUMED & 1.39 & 0.40 & 1.72 & 1.95 & 62.28 \\
\hline Reuters & 18.41 & 0.40 & 7.75 & 11.01 & 80.77 \\
\hline Sector-48 & 11.91 & 0.50 & 4.92 & 9.51 & 38.31 \\
\hline NewsGroup & 7.56 & 0.48 & 4.90 & 6.34 & 160.11 \\
\hline RCV1 & 10.46 & 0.42 & 4.64 & 6.79 & 38.54 \\
\hline
\end{tabular}

CPU time required by LibSvm is about 40 times larger than that of $\mathrm{MA}$ on OHSUMED and about 20 times larger on NewsGroup. In contrast to LibSvm, as a result, the time saving of MA is very obvious.

\subsection{Training Margin and Performance vs. MaxIteration}

Fig. 7 shows training margin and prediction performance curves of MA vs. MaxIteration on five datasets. Weight, LearnRate, and MinMargin are set to 0.2, 0.5, and 0.1 respectively; feature number is set to 10,000 .
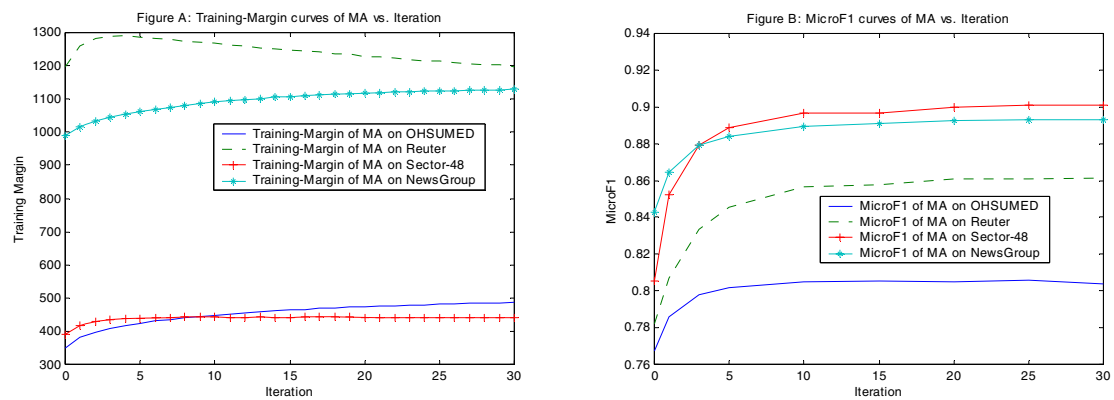

Fig. 7. Training margin and prediction performance of MA vs. MaxIteration

We can observe that proposed Model Adjustment can enlarge margin and boost prediction performance. This figure demonstrates that increasing the MaxIteration increases training margin and prediction performance. However, the increase in two measures is not directly proportional to increase in MaxIteration. As the MaxIteration is getting larger, the curves of two measures are starting to level off. 


\section{Conclusion Remarks}

In this work, a novel Model Adjustment (MA) algorithm was proposed to deal with model bias problem of Centroid Classifier. The basic idea is to pick out some training examples to adjust Centroid Classifier model. The main contributions are:

Firstly, in order to avoid over-train problem, we combine two measures for Model Adjustment: training-set errors and training-set margins. That is to say, misclassified examples as well as small-margin examples are picked out to update the classifier model.

Secondly, extensive experiments are conducted on five benchmark evaluation collections. The results show that Model Adjustment could make a significant difference on the performance of Centroid Classifier. Furthermore, the experimental results indicate margin can further improve the performance of Model Adjustment for Centroid Classifier.

\section{References}

1. Shankar, S., Karypis, G.: Weight adjustment schemes for a centroid based classifier. Technical report, Dept. of Computer Science, University of Minnesota

2. McCallum, A., Nigam, K.: A Comparison of Event Models for Naive Bayes Text Classification. In: AAAI/ICML-98 Workshop on Learning for Text Categorization, pp. 4148 (1998)

3. Mun, P.P.T.M.: Text Classification in Information Retrieval using Winnow, http://citeseer. ist.psu.edu/cs

4. Sebastiani, F.: Machine learning in automated text categorization. ACM Computing Surveys 34(1), 1-47 (2002)

5. Joachims, T.: Text categorization with support vector machines: learning with many relevant features. In: ECML, pp. 137-142 (1998)

6. Liu, Y., Yang, Y., Carbonell, J.: Boosting to Correct Inductive Bias in Text Classification. In: CIKM, pp. 348-355 (2002)

7. Wu, H., Phang, T.H., Liu, B., Li, X.: A Refinement Approach to Handling Model Misfit in Text Categorization. In: SIGKDD, pp. 207-216 (2002)

8. Tan, S., Cheng, X., Ghanem, M.M., Wang, B., Xu, H.: A Novel Refinement Approach for Text Categorization. In: ACM CIKM, Bremen, Germany, pp. 469-476 (2005) 